\title{
Investigation of Control Method for Connected-Automated Vehicle to Multiplex Dedicated Bus Lane
}

\author{
Wei-Jie Xiu $\left(\mathbb{D},{ }^{1}\right.$ Li Wang $\left(\mathbb{D},{ }^{1}\right.$ Meng-Yang Guo $\mathbb{D},{ }^{1}$ Li-Li Zhang $\mathbb{D}^{2},{ }^{2}$ and Qi Zhao ${ }^{1}$ \\ ${ }^{1}$ Beijing Key Laboratory of Urban Intelligent Traffic Control Technology, North China University of Technology, \\ Beijing 100144, China \\ ${ }^{2}$ College of Information Engineering, Beijing Institute of Petrochemical Technology, Beijing 102617, China \\ Correspondence should be addressed to Li Wang; li.wang@ncut.edu.cn and Li-Li Zhang; zhanglili@bipt.edu.cn
}

Received 27 August 2021; Revised 28 October 2021; Accepted 7 December 2021; Published 28 December 2021

Academic Editor: Peter Chen

Copyright (C) 2021 Wei-Jie Xiu et al. This is an open access article distributed under the Creative Commons Attribution License, which permits unrestricted use, distribution, and reproduction in any medium, provided the original work is properly cited.

Dedicated bus lanes (DBLs) have been widely utilized to ensure public transport priority. To improve overall road efficiency, various control methods of multiplexing DBL are developed and discussed. In this study, we focus on the control method which is based on the connected-automated vehicle (CAV) technology, and the proposed method is validated by using microscopic traffic simulation. The simulation results show that two proposed control methods of multiplexing DBL can reduce the average delay and the average number of stops and increase the travel speed. In comparison, the real-time control method based on the CAV technology offers better effects than the improved signal light control method.

\section{Introduction}

Under the conditions of the shortage of urban road resources and the high cost of infrastructure construction, one or more lanes have been set up on existing urban roads with traffic signs and markings as DBL for public buses, which has become one of the effective measures to ensure public transport priority in many big cities [1]. 89\% of cities in Europe, including cities which have smaller numbers of DBL, own DBL. Some large cities in China, including Beijing, Shanghai, Guangzhou, Shenzhen, Qingdao, Kunming, Hangzhou, Wuhan, Xi'an, Shijiazhuang, and so on, have also built a large number of DBLs [2].

Although DBL can improve bus operation efficiency, there are two main problems: one is the reduction of total road capacity and the other is the low utilization rate of DBL, leading to a waste of road resources. Numerous research studies have attempted to multiplex these road resources, which might be potentially wasted. A commonly used method is to restrict the time for DBL so that only buses can use them during certain periods of the day (morning and evening peak hours), and other vehicles are not allowed to enter during these periods, and during the rest of the time, the DBL is used as a general lane for all vehicles. However, this solution does not take into account dynamic traffic demands of road resources for various types of vehicles. When other vehicles are prohibited from entering the DBL, there are still some spare road resources that could be utilized; during the period of mixed use of buses and other types of vehicles on DBL, the lack of effective control measures for other types of vehicles is easy to affect the efficiency of bus system, and the public transport priority cannot be guaranteed. Viegas first proposed the concept of intermittent bus lanes (IBLs), which determine whether a certain section of the lane becomes a DBL according to whether the bus presents or not: when the bus approaches a certain section of the lane, this lane becomes a DBL; when the bus leaves this section, the lane becomes a normal lane and is open to all types of vehicles $[3,4]$. Eichler et al. found that bus lanes with intermittent priority (BLIPs), unlike dedicated ones, do not significantly reduce street capacity [5].

With the advanced technologies of vehicle to everything (V2X), Internet of vehicle (IOV), and CAV, the control method and the logic of designing DBL can be improved 
dramatically. For instance, the roadside units can be set up near the bus lane to collect and upload the real-time bus positioning data and traffic operation data to the central control platform and then the dynamic control strategy for different vehicles could be implemented by combining the the real-time data and the control algorithm [6].

In practical situations, CAV technology will firstly be used in commercial vehicles [7], which include buses, freight vehicles (logistic, express delivery, and so on), etc. What is strikingly noticeable is that the operation characteristics (speed and acceleration) of these vehicles are relatively similar. The mixed use by slow-moving vehicles and fast-moving vehicles is one of the most important reasons that limit the road capacity to reach the designed level [8], and slow-moving vehicles, such as buses or trucks, will cause "movement bottleneck" phenomenon $[9,10]$. A possible scenario in the near future is that the commercial vehicles have already popularized CAV technologies, while other types of vehicles are still in a state of human driving. In this case, considering to multiplex the DBL for these slow-moving vehicles would have the following three advantages: the first is that multiplexing DBL will reduce the occurrence of moving bottleneck [11]; the second is that the efficiency of the DBL under the premise of ensuring public transport priority will be improved; the third is that the CAV freight vehicles can be investigated as the basis of DBL multiplexing technology, which is favourable for the efficiency of DBL.

Zhu [12] compared DBL with IBL by using a cellular automaton traffic flow model, and the results proved that when the traffic flow was low, opening the DBL to general vehicles significantly improved the efficiency of road traffic. Zyryanov and Mironchuk [13] used the microscopic traffic simulation model to evaluate the effects of different traffic volume and bus priority signals on the IBL and found that the IBL can increase the travel speed of public buses and other vehicles even when the traffic volume increased. $\mathrm{Wu}$ et al. [14] explored the benefits of BLIP. Yang and Wang [15] compared the influences of DBL and IBL on the buses and adjacent traffic, and the results showed that IBL performs better than DBL because it has less negative impact on other vehicles. Joskowicz [16] studied the following issues: the configuration method of dynamic traffic signs on the IBL, the data collection, the queuing time of public buses on the IBL, and the effect of IBL on road capacity. Dong and Zhao [17] developed a "time division multiple" method of DBL which can calculate the appropriate time period for other vehicles to "borrow" the bus lane. Chiabaut and Barcet [18] assessed the impact of IBL on the public traffic and suggested that the IBL system could be a promising strategy; when it is combined with the traffic signal priority, the average bus travel time is significantly reduced. Song et al. [19] suggested a shared lane of DBL and right-turn-exclusive lane to reduce the impact of DBL on traffic conditions.

The studies mentioned above have focused on the impact of multiplexing DBL on the traffic flow, and the effect of different types of vehicles as the main multiplexing subject was neglected; moreover, the control device such as traffic signal is the main DBL multiplexing control device, and the limitations of such device and method also affect the performance of DBL multiplexing. If CAV and other advanced technology can be applied to DBL multiplexing, the application effect of IBL can be enhanced at a lower cost while ensuring bus priority.

Regarding the highly controllable and self-control achievable character of CAVs, which are similar to buses, this study takes the CAVs as the DBL utilization subject. On the basis of the existing multiplexing control method, the new method which combines signal light and CAV technology is proposed. Then, the traffic simulation will be used to evaluate and compare the impact of the two implementation methods on the road traffic [20]. Considering the need of secondary development and research conditions in this study, we select PTV Vissim and Python as the traffic simulation tools.

\section{Control Methods of DBL Multiplexing}

2.1. Method 1: Improved Signal Light Control Method. In this method, as the signal detection and control device, the roadside units were set from the upstream intersection beside the DBL at intervals of distance $L$, the position and speed of CAVs and buses were collected in real time, and they were uploaded to the central control platform. The platform returned the control signal to the roadside units according to the control strategy. The CAVs thus followed the instruction of roadside units to enter or exit the DBL. Figure 1 shows the sketch map of this method. The control strategy was divided into flowing five steps [17].

(1) Taking the $i$-th roadside unit as the center of a circle and using $R$ as the radius to search for the closest bus to the upstream of the unit, which is called the approaching bus, the distance to the $i$-th roadside unit is $D_{\text {bus }}(i)$ (according to the distance to the upstream intersection, from near to far, $i=1,2, \ldots, n$, where $n$ is the total number of roadside units on the utilizing road section).

(2) Assuming that the CAV is allowed to travel on the DBL between the $i$-th and the $(i+1)$-th roadside unit at the moment $t$, the roadside unit $i$-th will broadcast the signal "CAVs are allowed to enter," and the holding time of this signal is the DBL multiplexing time; at the end time, the roadside unit $i$-th will broadcast the signal "CAVs are prohibited from entering," at the same time, CAVs on the DBL must exit.

(3) The travel time of the CAV between $i$-th and $(i+1)$-th units is calculated as

$$
T_{\text {cav }}=\frac{L}{v_{\text {cav }}}+T_{\text {borrow }}+\lambda_{1} T_{\text {light }}+T_{\text {return }}
$$




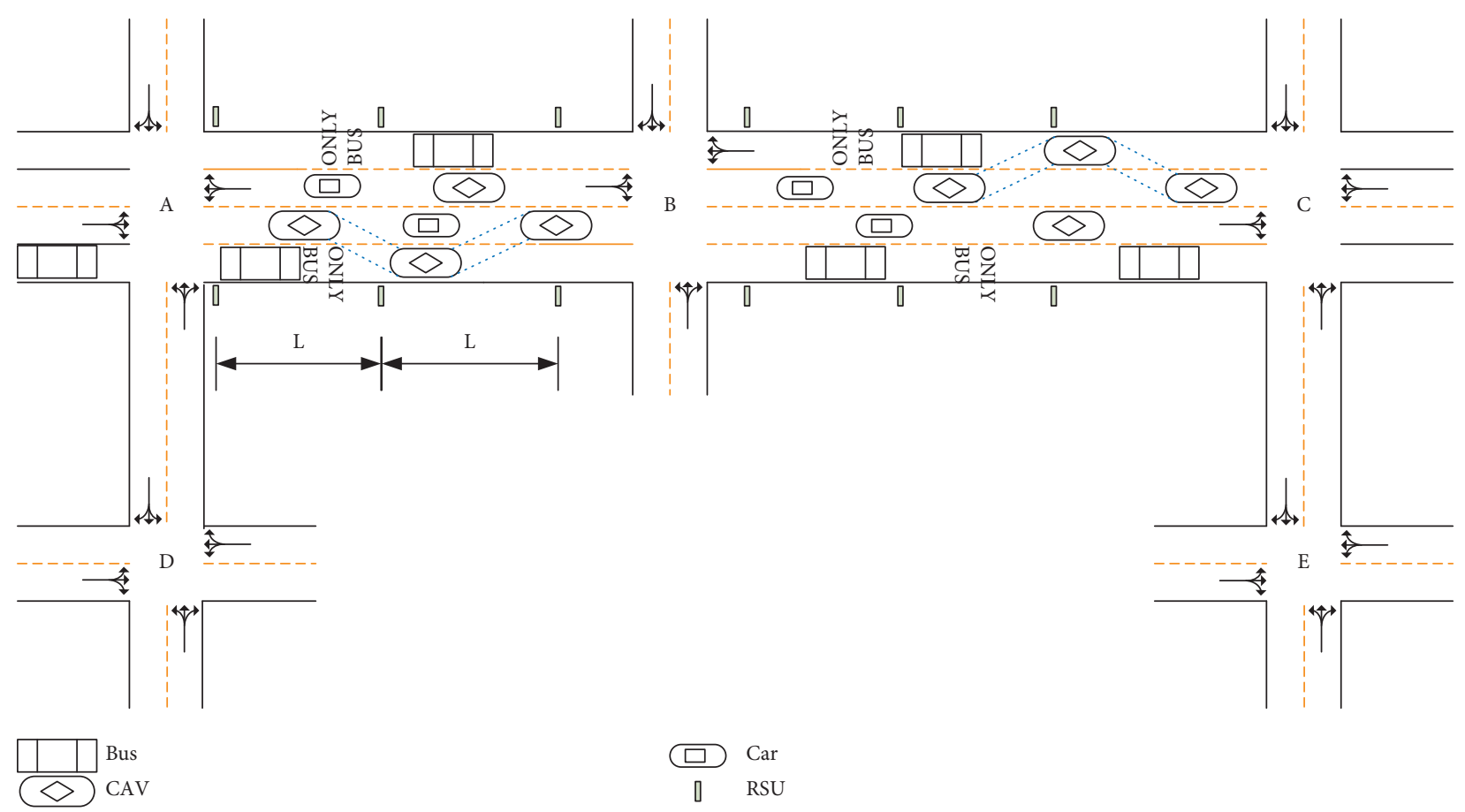

Figure 1: The sketch map of method 1.

where $T_{\text {light }}$ is the predicted waiting time for the signal lights at the downstream intersection; $v_{\text {cav }}$ is the speed of the CAV; $T_{\text {borrow }}$ is the minimum time required for the CAV to enter the DBL; $T_{\text {return }}$ is the minimum time required for the $\mathrm{CAV}$ to exit the DBL; $T_{\text {borrow }}$ and $T_{\text {return }}$ are affected by many factors such as individual driver differences, roads, and weather; and $\lambda 1$ indicates the introduced coefficient, assuming that there are two following scenarios: when there is no intersection between $i$-th and $(i+1)$ th units, $\lambda_{1}=0$; when there is an intersection, $\lambda_{1}=1$.

(4) The travel time of the approaching bus from the current position to the $(i+1)$-th unit searched at the $i$-th unit is calculated as

$$
T_{\text {bus }}=\frac{D_{\text {bus }}(i)+L}{v_{\text {bus }}}+\lambda_{2} T_{\text {light }} \text {, }
$$

where $v b u s$ is the speed of the approaching bus; $\lambda_{1}$ indicates the introduced coefficient, assuming that there are two following scenarios: when there is no intersection between $i$-th and (i+1)-th units, $\lambda_{1}=0$; when there is an intersection, $\lambda_{1}=1$.

(5) Determine whether to allow CAVs to multiplex DBL.

The methodology is further illustrated through the use of two scenarios.

The first scenario is to take the $i$-th roadside unit as the center of a circle and use $R$ as the radius to search for the closest bus to the upstream of the unit. If no approaching bus is found, the CAV will be allowed to enter the DBL between units $i$-th and $(i+1)$-th, the roadside unit $i$-th will broadcast the signal "CAVs are allowed to enter DBL."
The second scenario is to take the $i$-th roadside unit as the center of a circle and use $R$ as the radius to search for the closest bus to the upstream of the unit. If the approaching bus is located, the formula is

$$
T_{\text {bus }} \geq T_{\text {cav }}+T_{\text {min }}+T_{\text {head }},
$$

where $T_{\text {head }}$ is the minimum headway between the CAV and the bus on DBL (specified as 1.5 seconds to 2 seconds) (defined by numerous studies) and $T_{\min }$ is the minimum time for the CAV using the DBL (too short will cause frequent lane changing, whereas too long will affect the bus priority). Figure 2 shows the flowchart of this method.

\subsection{Method 2: Real-Time Control Method Based on CAV} Technology. In this method, the real-time position and speed data of CAVs is known, taking the CAV as the center of a circle, $R$ as the radius, according to whether there is an approaching bus within the search radius, comparing the moving characteristics of the approaching bus with the CAV to decide whether the CAV is allowed to enter the DBL, the steps are as follows. Figure 3 shows the sketch map of this method.

(1) If there is no approaching bus in the searching area, the CAV is allowed to enter the DBL.

(2) If there is one and only bus in the searching area, it is necessary to judge whether the CAV is allowed to enter or is forced to leave. The judgment method is as follows:

$$
T_{\text {borrow }} \leq T_{\text {bus }}-T_{\text {head }}=\frac{D_{\text {bus }}}{v_{\text {bus }}}-T_{\text {head }},
$$




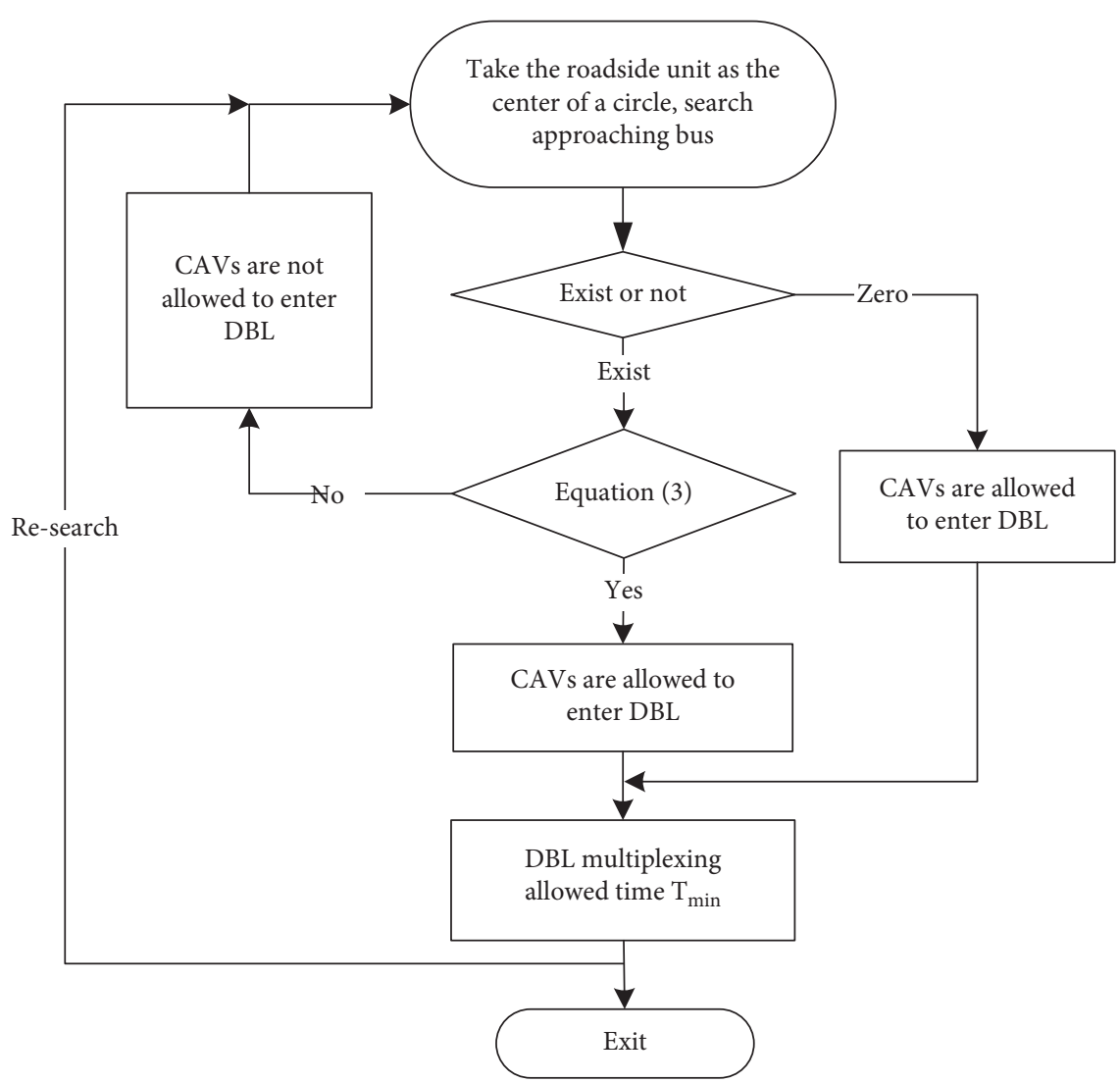

FIGURE 2: The flowchart of method 1.

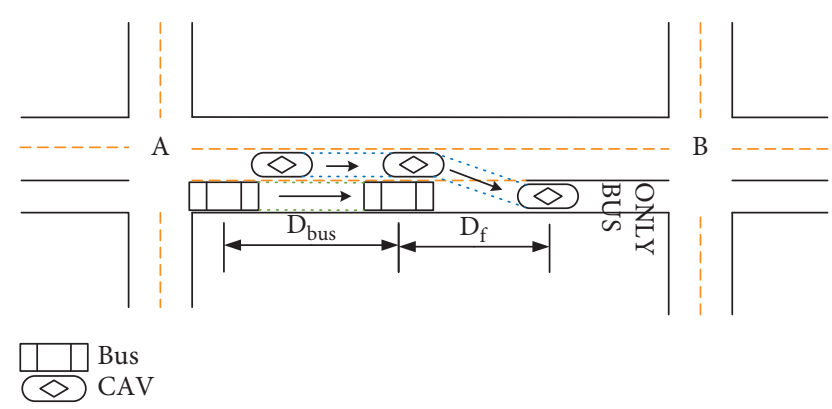

FIgURE 3: The sketch map of method 2.

where $T_{\text {borrow }}$ is the minimum time required for the CAV to enter the DBL; $T_{\text {bus }}$ is the time required for the bus to travel from the current position to the safe headway with the smallest distance from the CAV on the DBL; $T_{\text {head }}$ is the minimum headway safety time when the vehicle changes lanes; $D_{\text {bus }}$ is the distance from the bus's current position to the headway safety position of the CAV on the DBL; and $v_{\text {bus }}$ is the speed of the bus.

(3) If there are multiple buses in the searching area, the closest bus is determined as an approaching bus. Since the premise of multiplexing DBL is to ensure the bus priority and not cause interference to the buses, if there are multiple buses within the search radius, it is considered that the duration of multiplexing the DBL must exceed all the buses within the search radius. The judgment method is as follows:

$$
T_{\text {borrow }} \leq T_{\text {dsbus }}-T_{\text {head }}=\frac{D_{\text {dsbus }}}{v_{\text {dsbus }}}-T_{\text {head }},
$$

where $T_{\mathrm{dsbus}}$ is the time required for the closest bus to travel from the current position to the safe headway with the smallest distance from the CAV on the DBL; $T_{\text {head }}$ is the minimum headway safety time when the vehicle changes lanes; $v_{\mathrm{dsbus}}$ is the speed of the bus; and $D_{\mathrm{dsbus}}$ is the distance from the bus's current position to the headway safety position of the CAV on the DBL. Figure 4 shows the flowchart of this method.

\section{Simulation Experiment Design}

In this study, Vissim and Python were used to verify and compare the impact of the two DBL multiplexing control methods mentioned above on traffic flow.

3.1. Objective Function. The travel speed and road capacity are important indices of road performance evaluation. This study takes the travel speed of the CAV $\bar{V}_{\text {cav }}$ as the optimization goal.

$$
F\left(R, T_{\text {min }}\right)=\operatorname{Min}\left(\bar{V}_{\text {cav }}\right) .
$$




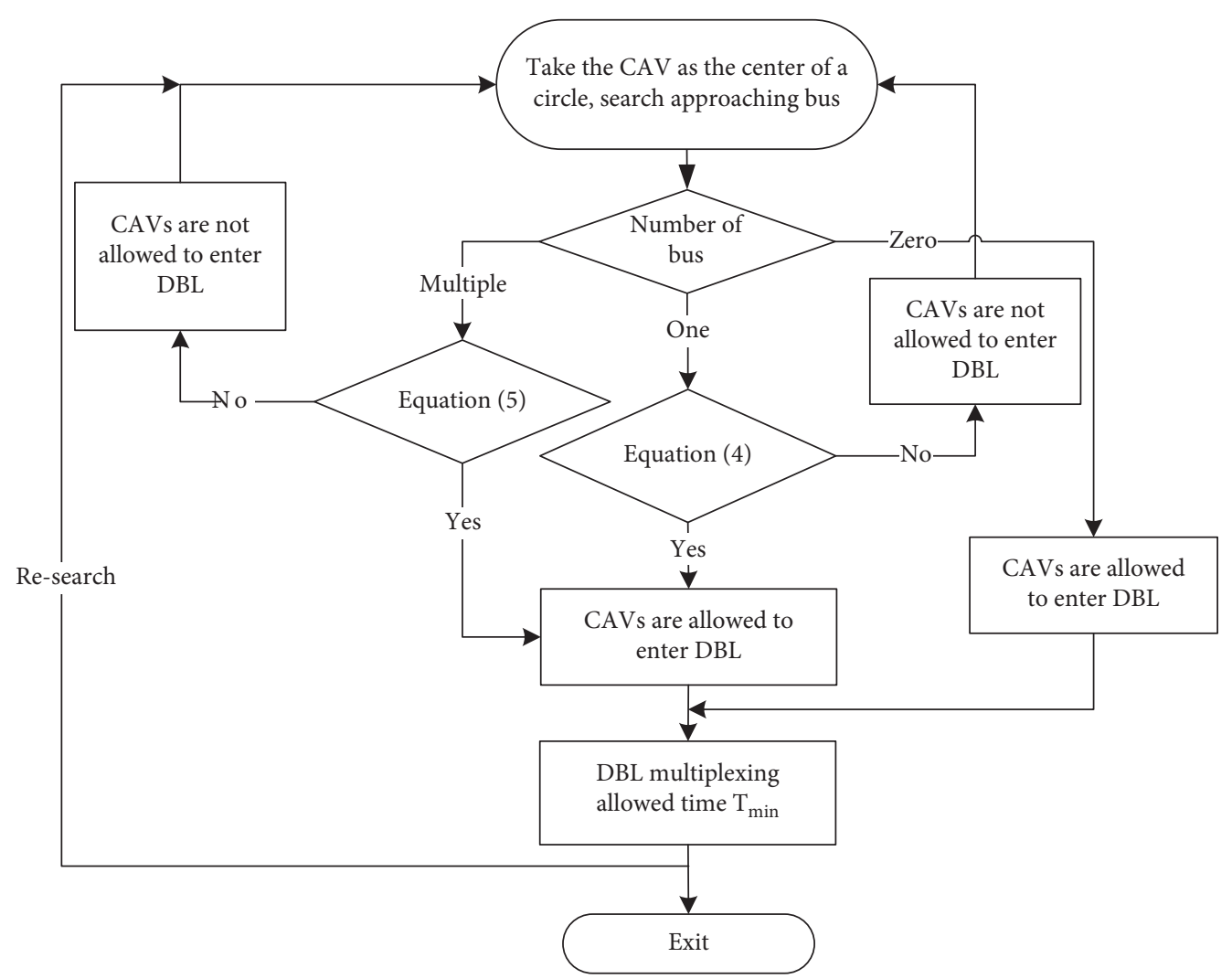

Figure 4: The flowchart of method 2.

\subsection{Constraints}

3.2.1. Bus Volume. The purpose of setting up DBL is to ensure bus priority. Compared with normal lanes, buses can get a better driving environment with DBL. When the bus traffic volume on the bus lane is greater than the volume on the adjacent normal lane, the driving environment of the bus on the DBL is actually inferior to that of the normal lane, and the bus lane loses its multiplexing significance. The following constraints must be met between the bus flow and other vehicles' flow:

$$
Q_{\text {bus }}<\frac{Q_{\text {lane }}}{n_{1}},
$$

where $Q_{\text {bus }}$ is the bus volume of the DBL; $Q_{\text {lane }}$ is other vehicles volume; and $n 1$ is the quantity of normal lanes in the road section.

3.2.2. Saturation of $D B L$. According to the U.S. "Highway Capacity Manual" and the literature research summary [21], when the saturation on the DBL is greater than 0.7 , the lane will be congested, the effect of multiplexing DBL will decrease sharply, and the bus delay will significantly increase. In this research, the following saturation constraint was set:

$$
\frac{Q_{\text {bus }}+Q_{\text {cav }}}{C_{\text {bus }}}<0.7
$$

where $Q_{\text {bus }}$ is the bus volume of the $D B L ; Q_{\text {car }}$ is the CAV volume of multiplexing $\mathrm{DBL}$; and $C_{\text {bus }}$ is the capacity of the DBL.

\section{Simulation Results and Analysis}

A road network in Shijingshan District, Beijing, is established in the Vissim, as shown in Figure 5. The chosen experimental road section is $2,300 \mathrm{~m}$ long from east to west, and the nearside lane is the DBL. The experimental road section is a long-distance road with four intersections. Seven roadside units are set next to the DBL: three roadside units are set on the east of intersection 1, with distance of $100 \mathrm{~m}$, $400 \mathrm{~m}$, and $700 \mathrm{~m}$, respectively; two roadside units are set on the east of intersection 2, with distance of $50 \mathrm{~m}$ and $300 \mathrm{~m}$; one roadside unit is set on the east of intersection 3 , with distance of $80 \mathrm{~m}$; and one roadside unit is set on the south of intersection 4 , with distance of $50 \mathrm{~m}$.

Figures 6-8 show the phase and time of the four intersections on the experimental road; the simulation time is $3,600 \mathrm{~s}$; the time required for the CAV to enter the DBL, $T_{\text {borrow }}$, is $15 \mathrm{~s}$; the time required for the CAV to exit the $\mathrm{DBL}, T_{\text {return }}$, is $12 \mathrm{~s}$; the minimum safety headway time during lane changing, $T_{\text {head }}$, is $2 \mathrm{~s}$; the minimum time for using the DBL, $T_{\min }$, is $30 \mathrm{~s}$ [22]; and the search radius, $R$, is $20 \mathrm{~m}$. Considering the background of multiplexing DBL is the road traffic has reached saturation, the single lane input volume is set as $q=1,800 \mathrm{pcu} / \mathrm{h}$. The average delay, average 


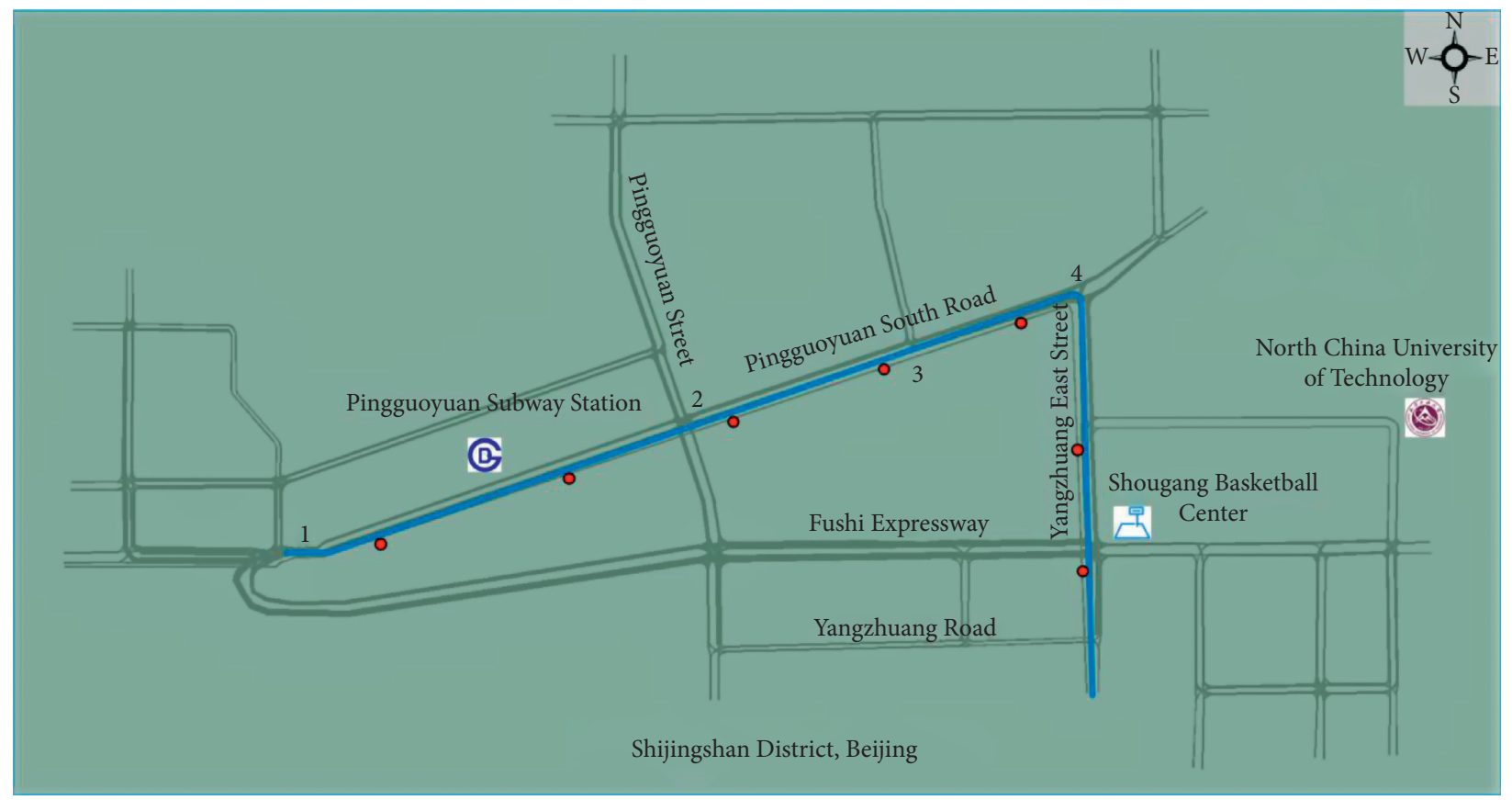

- Bus Stop

- Bus Lane

FIgure 5: The traffic simulation road network.

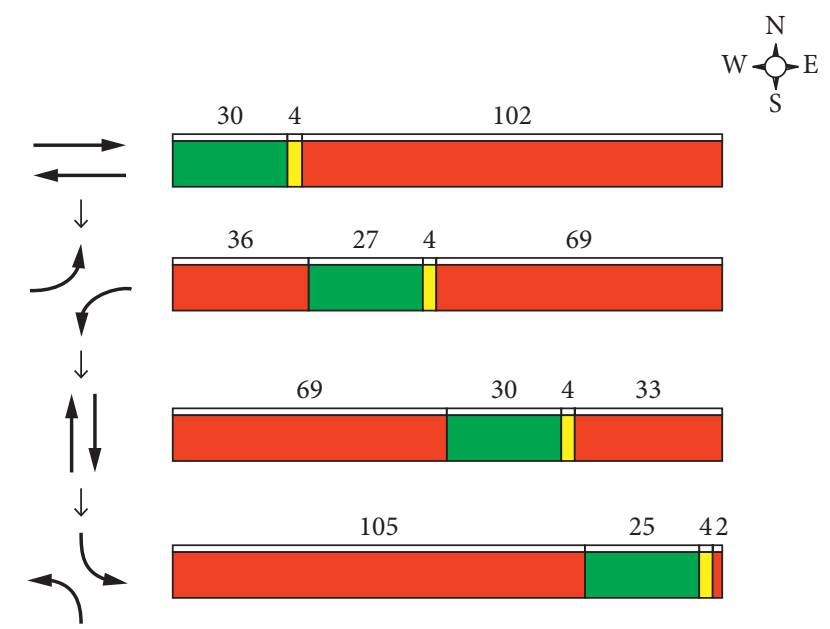

FIgURE 6: The phases and duration time of intersection 1 and intersection 2 .

number of stops, and travel speed are selected as the indicators for the effect evaluation of multiplexing DBL.

Three simulation experiments were conducted: normal use of DBL (normal), multiplexing DBL by CAV controlled by signal light (method 1), and multiplexing DBL by CAV controlled by real-time situation (method 2). Figures 9-11 show the average delay, the average number of stops, and the travel speed of different vehicles in the three situations.

The simulation results showed that the two control methods were implemented under the premise of bus priority. Both method 1 and method 2 can reduce the delay of CAVs and other vehicles most of the time.
As shown in Figure 9(b), method 2 had a greater effect on the delay reduction of CAVs than method 1 . The reason was that method 2 is more flexible in real-time monitoring and judgment of the vehicle position than the roadside unit signal light in method 1. Figure 9(a) shows that both methods increase the average delay of other vehicles at specific time. The merging lanes of CAVs had impacted on other general vehicles.

Figure 10 shows that under the premise of the average number of bus stops did not significantly increase, both methods can reduce the number of stop times of CAVs and other vehicles most of the time, while method 2 performed better than method 1 .

Through the analysis and verification of visualized data, the results in Figure 11 showed that both method 1 and method 2 have improved the travel speed of CAVs and other vehicles, and the priority of buses was not intervened.

During the simulation experiment period, the travel speed of CAVs and other vehicles without control methods was $44.7 \mathrm{~km} / \mathrm{h}$ and $36.4 \mathrm{~km} / \mathrm{h}$, respectively. In method 1 , the travel speed of CAVs and other vehicles was $47.3 \mathrm{~km} / \mathrm{h}$ and $37.8 \mathrm{~km} / \mathrm{h}$, respectively, whereas the numbers were $48.8 \mathrm{~km} /$ $\mathrm{h}$ and $39.9 \mathrm{~km} / \mathrm{h}$ in method 2. Compared with the normal situation, method 1 increased the travel speed of normal vehicles and CAVs by $5.8 \%$ and $3.8 \%$, respectively, and method 2 increased that of normal vehicles and CAVs by $9.2 \%$ and $9.6 \%$, respectively. The improvement effect of method 2 was clearly greater than that of method 1 . The reason was that the real-time detection used by method 2 was more flexible than the roadside unit signal light control, 


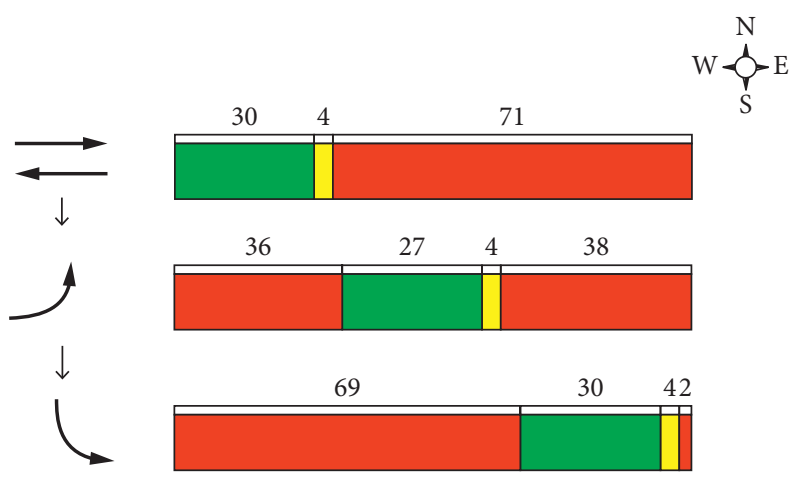

FIgURE 7: The phases and duration time of intersection 3.

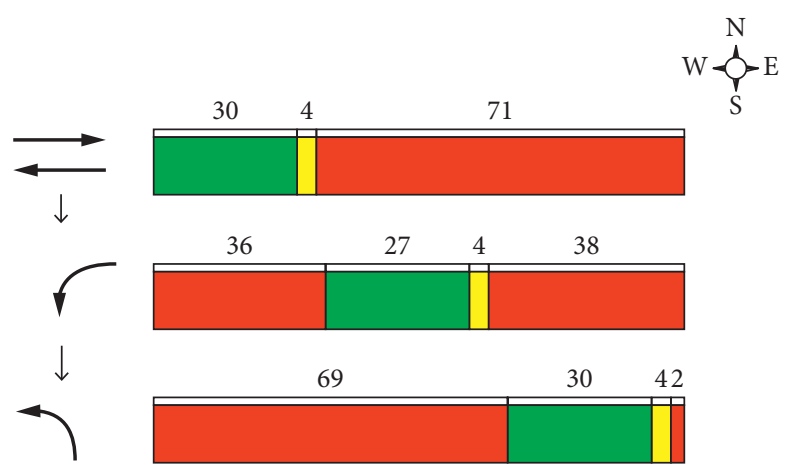

FIgURE 8: The phases and duration time of intersection 4.

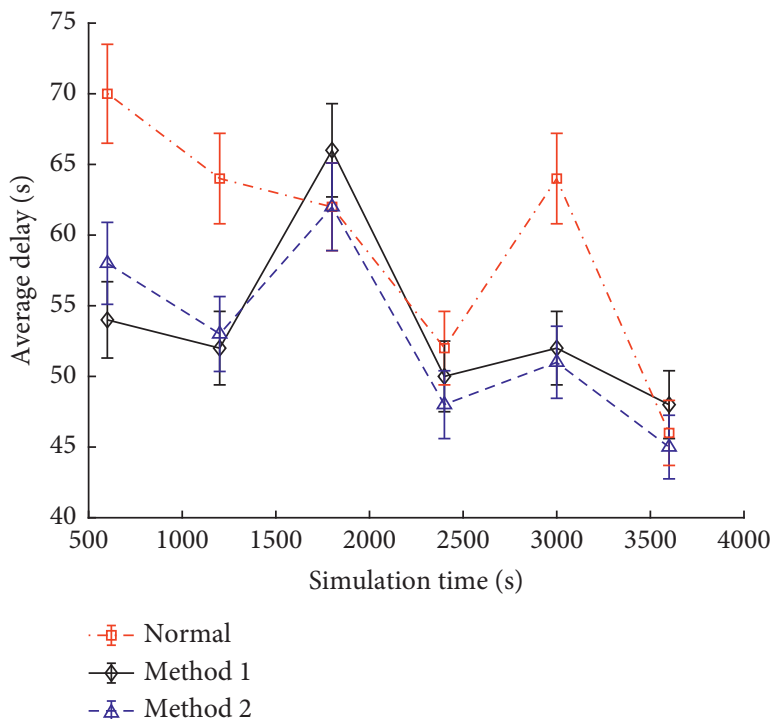

(a)

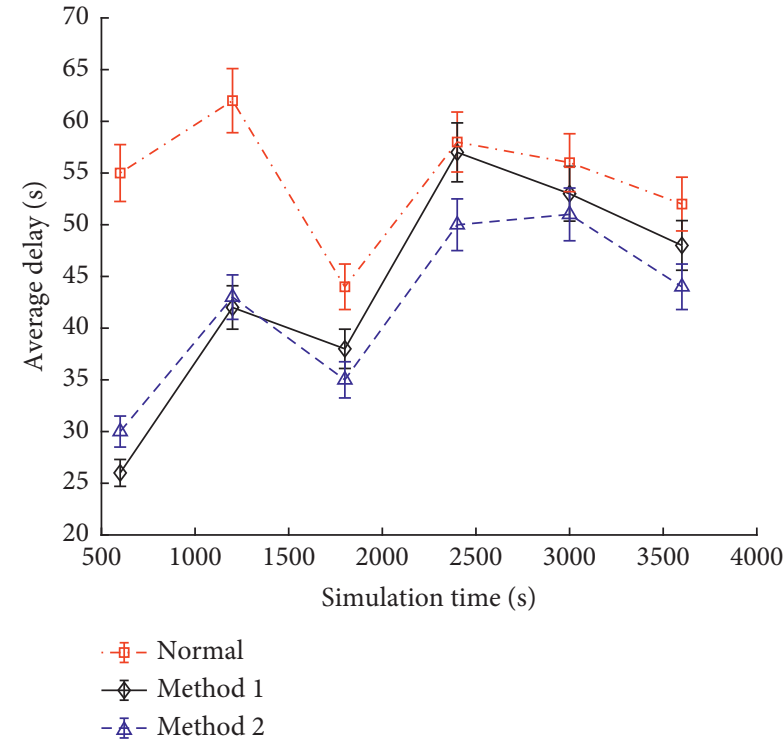

(b)

Figure 9: Continued. 


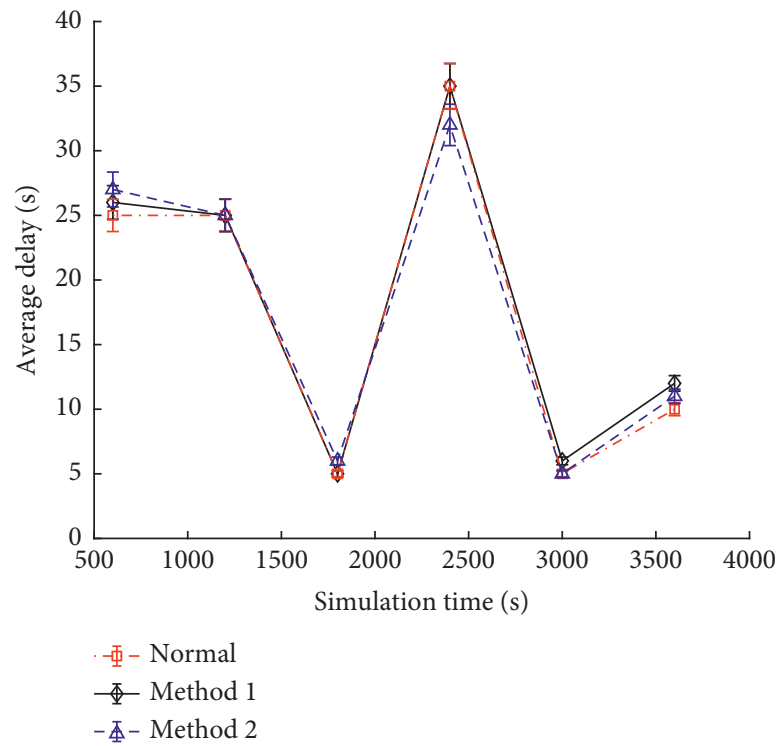

(c)

Figure 9: Average delay of different vehicles in the three situations. (a) Average delay of general vehicles in the three situations. (b) Average delay of CAVs in the three situations. (c) Average delay of buses in the three situations.

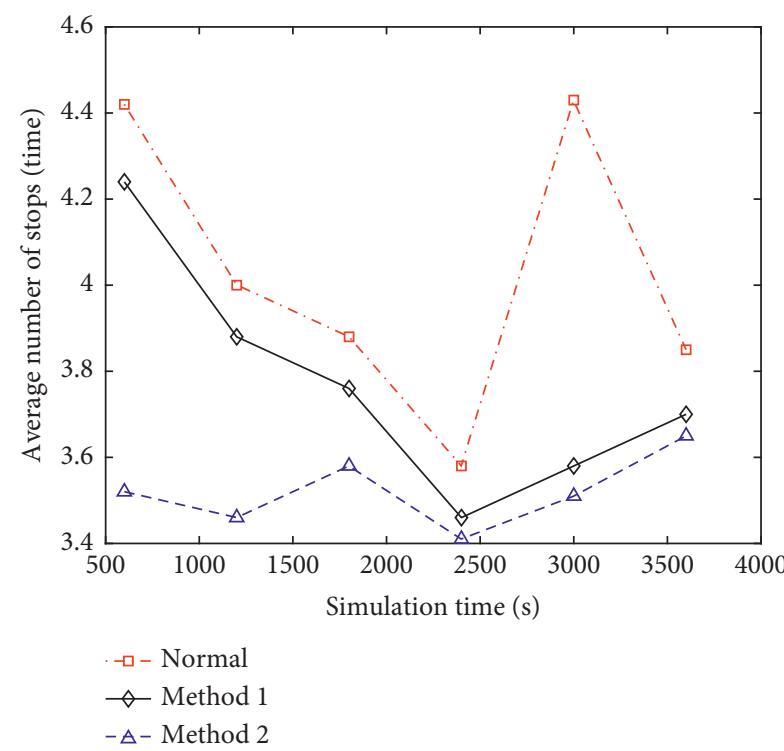

(a)

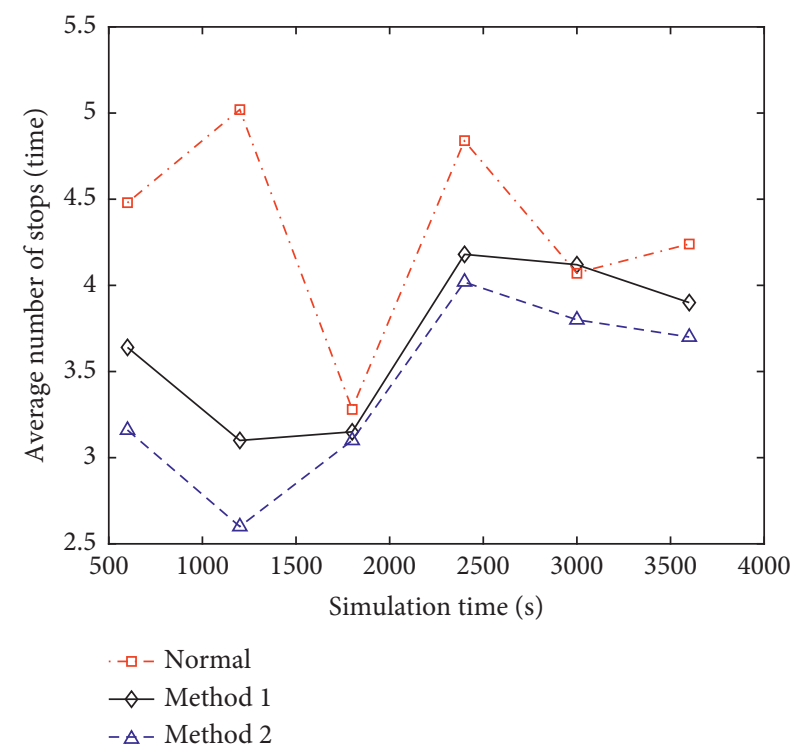

(b)

Figure 10: Continued. 


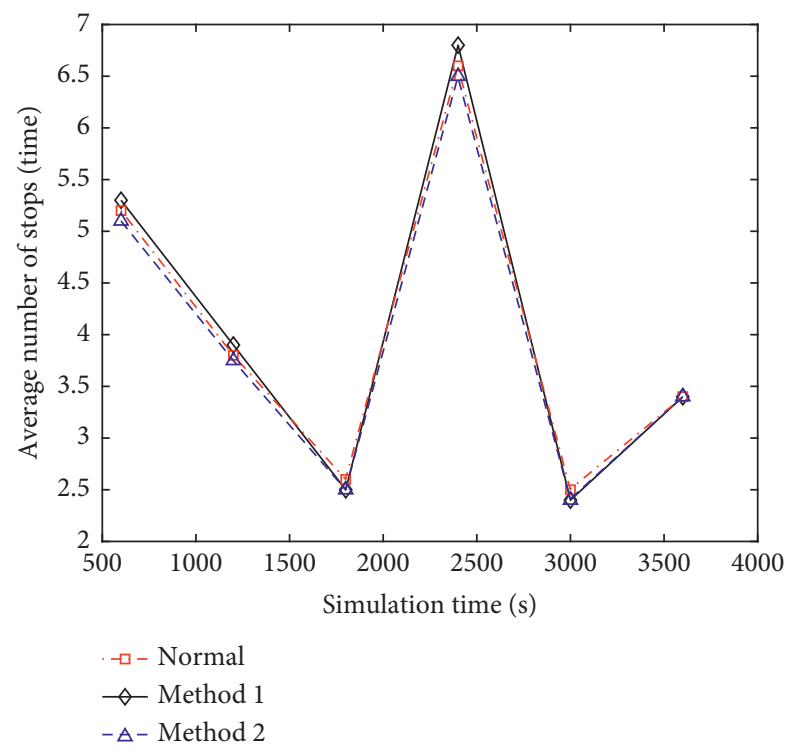

(c)

Figure 10: Average number of stops of different vehicles in the three situations. (a) Average number of stops of general vehicles in the three situations. (b) Average number of stops of CAVs in the three situations. (c) Average number of stops of buses in the three situations.

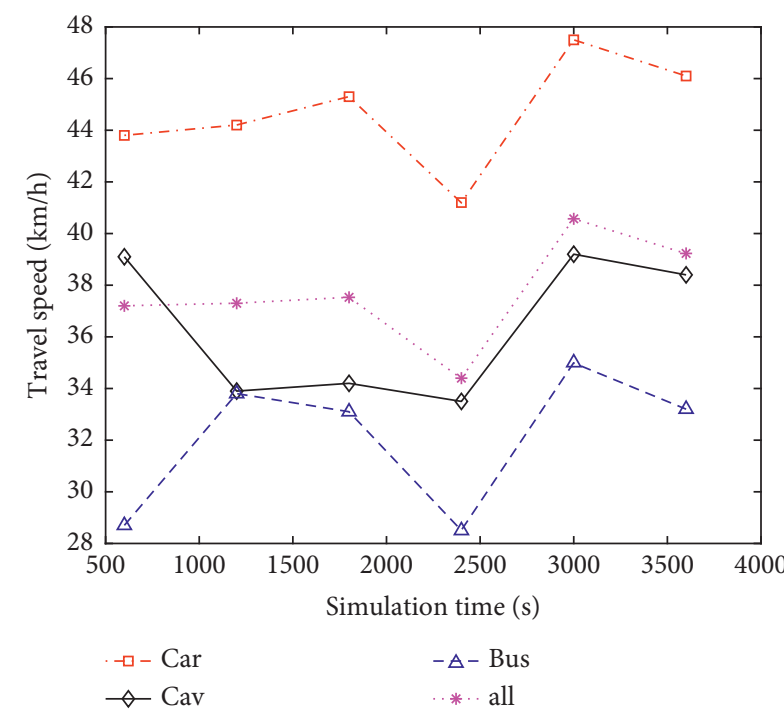

(a)

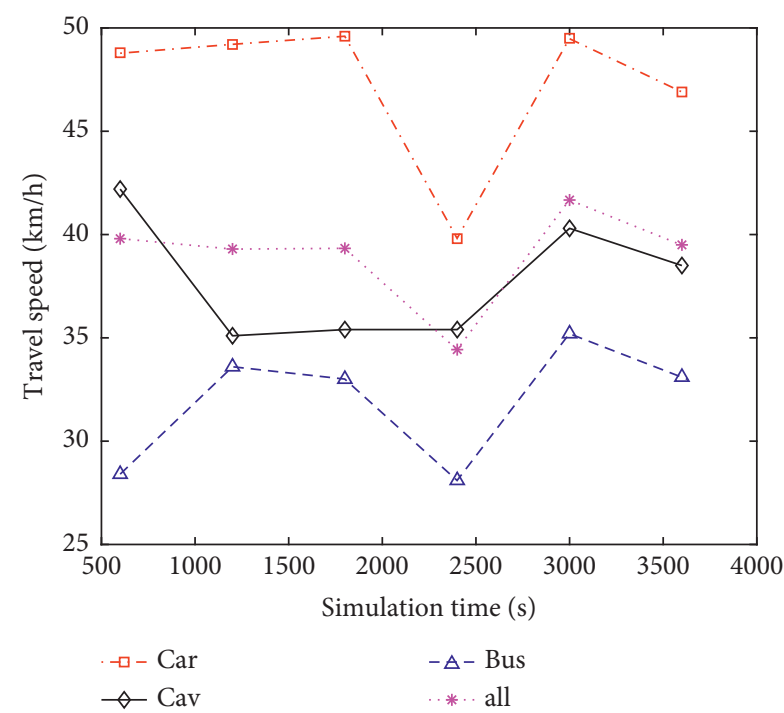

(b)

Figure 11: Continued. 


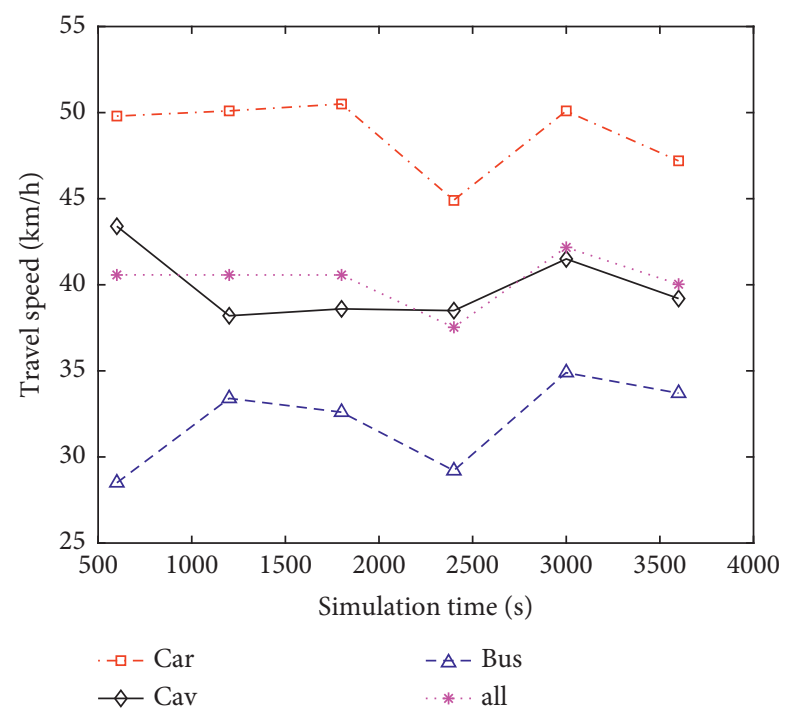

(c)

Figure 11: Travel speed of different vehicles in the three situations. (a) Travel speed of different vehicles in normal control situation. (b) Travel speed of different vehicles in method 1. (c) Travel speed of different vehicles in method 2.

and method 2 maximized the use of road resources under the premise of ensuring the priority of buses.

\section{Conclusion}

This paper investigated the multiplexing of DBL for CAVs through the microscopic traffic simulation model; two different control methods were implemented under the same road condition, and traffic volume was analyzed. The results indicated that

(1) Both methods could improve the driving performance of CAVs and other vehicles, and the efficiency of DBL and the capacity of roads are increased under the premise of ensuring bus priority.

(2) The real-time control method based on the CAV technology is more efficient than the improved signal light control method, which fully shows that with the development of the CAV technology, the method with CAVs as the main control subject will be more flexible and effective.

(3) The multiplexing DBL control method for CAVs proposed in this paper can be used as a transition plan from the existing signal light control method to the future application of CAVs. The future study will mainly focus on two parts: exploring the traffic flow characteristics of mixed CAVs and human-driving vehicles; investigating the effects of DBL multiplexing on the bus priority signal control.

\section{Data Availability}

The data used to support the findings of this study are available from the corresponding authors upon request only for research purpose.

\section{Conflicts of Interest}

The authors declare that they have no conflicts of interest.

\section{Acknowledgments}

This research was funded by the Beijing Natural Science Foundation (nos. 4214070 and 4194078), Beijing Municipal Great Wall Scholar Program (CIT\&TCD20190304), CrossDisciplinary Science Foundation from Beijing Institute of Petrochemical Technology (project no. BIPTCSF-006), 2021-2023 Young Talents Promotion Project of Beijing Association for Science and Technology, and Open Project for Beijing Urban Governance Research Base of North China University of Technology (no. 21CSZL34).

\section{References}

[1] Y. Luo and D. Qian, "Impact scope of bus passenger flow caused by bus lane construction," Journal of Harbin Institute of Technology, vol. 51, no. 03, pp. 120-126, 2019.

[2] Y. Zhao, Research on Bus Lane Utilizing Technology and Vehicle Fractal Cooperation Method, Zhejiang University of technology, Hangzhou, China, 2011.

[3] J. Viegas, "Turn of the century, survival of the compact city, revival of public transport," in Transforming the port and transportation business, H. Meersman and E. Van de Voorde, Eds., pp. 51-63, Uitgeverij Acco, Antwerp, Belgium, 1997.

[4] J. Viegas and B. Lu, "The Intermittent Bus Lane signals setting within an area," Transportation Research Part C: Emerging Technologies, vol. 12, no. 6, pp. 453-469, 2004.

[5] M. Eichler and C. F. Daganzo, "Bus lanes with intermittent priority: strategy formulae and an evaluation," Transportation Research Part B: Methodological, vol. 40, no. 9, pp. 731-744, 2006.

[6] J. Song, X. Yang, Z. Cui, and C. Zhou, "Research on time division multiplexing system for bus lanes based on vehicleroad coordination and vehicle-to-vehicle communication," 
Information Recording Materials, vol. 020, no. 007, pp. 195198, 2019.

[7] Y. Lun, "Development status and trend of autonomous driving industry," Telecommunications Network Technology, vol. 6, no. No.276, pp. 40-43, 2017.

[8] H. Lin, Q. Fu, and H. Zhang, "Moving bottleneck theorybased new countermeasure for influence of heavy vehicles," Journal of Tongji University: Natural Science, vol. 35, no. 09, pp. 1209-1213, 2007.

[9] D. C. Gazis and R. Herman, "The moving and "phantom" bottlenecks," Transportation Science, vol. 26, 1992.

[10] C. Lattanzio, A. Maurizi, and B. Piccoli, "Moving bottlenecks in car traffic flow: a PDE-ODE coupled model," SIAM Journal on Mathematical Analysis, vol. 43, no. 1, pp. 50-67, 2011.

[11] G. Piacentini, P. Goatin, and A. Ferrara, "Traffic control via moving bottleneck of coordinated vehicles," IFAC-PapersOnLine, vol. 51, no. 9, pp. 13-18, 2018.

[12] H. B. Zhu, "Numerical study of urban traffic flow with dedicated bus lane and intermittent bus lane," Physica A: Statistical Mechanics and Its Applications, vol. 389, no. 16, pp. 3134-3139, 2010.

[13] V. Zyryanov and A. Mironchuk, "Simulation study of intermittent bus lane and bus signal priority strategy," Procedia - Social and Behavioral Sciences, vol. 48, no. 1, pp. 1464-1471, 2012.

[14] W. Wu, L. Head, W. Ma et al., BLIP: Bus Lanes with Intermittent Priority, Transportation Research Board Meeting, Washington, D C, 2013.

[15] H. Yang and W. Wang, "An innovative dynamic bus lane system and its simulation-based performance investigation," in Proceedings of the IEEE Intelligent Vehicles Symposium, pp. 105-110, Xian, June 2009.

[16] I. F. Joskowicz, Dynamic Bus Lane, The University of Texas at Arlington, Arlington, Texas, 2012.

[17] H. Dong and Y. Zhao, Optimization of Time Division Multiplexing for Dedicated Bus Lanes Based on Paramics, Journal of Zhejiang University of Technology, no. 01, pp. 65-69, China, 2012.

[18] N. Chiabaut and A. Barcet, "Demonstration and evaluation of an intermittent bus lane strategy," Public Transport, vol. 11, no. 1, pp. 443-456, 2019.

[19] X. Song, L. Ma, L. Li, Y. Gao, and M. Liu, "Simulation analysis and benefit evaluation of bus and right turn exclusive lanes," China Journal of Highway and Transport, vol. 32, no. 05, pp. 146-156, 2019.

[20] H. Xu, Q. Zheng, and K. Zhang, "Arterial road BRT signal priority method based on vertical equality," China Journal of Highway and Transport, vol. 32, no. 001, pp. 144-153, 2019.

[21] National Research Council (U.S), HCM2010: Highway Capacity Manual, Transportation Research Board, Washington, D.C., 5th ed edition, 2010.

[22] J. Yang, Research on the Impact of Frequent Lane Changes on Road Efficiency and Safety, Chang'an University, Xian, China, 2016. 